Z. Wahrscheinlichkeitstheorie verw. Gebiete $43,183-185$ (1978)
Zeitschrift für

Wahrscheinlichkeitstheorie und verwandte Gebiete

(C) by Springer-Verlag 1978

\title{
An Inequality for the Weights of Two Families of Sets, Their Unions and Intersections
}

\author{
Rudolf Ahlswede ${ }^{1}$ and David E. Daykin ${ }^{2}$ \\ 1 Fakultät für Mathematik, Universität 4800 Bielefeld, Federal Republic of Germany \\ 2 Department of Mathematics. University of Reading, Whiteknights, \\ Reading, Berkshire, England
}

\section{Introduction}

The object of this note is to prove

Theorem 1. Let $S$ be the family of all subsets of the set $\{1,2, \ldots, n\}$. If $\alpha, \beta, \gamma, \delta$ are non-negative real valued functions on $S$ such that

$$
\alpha(a) \beta(b) \leqq \gamma(a \cup b) \delta(a \cap b) \quad \text { for all } a, b \in S,
$$

then

$$
\alpha(A) \beta(B) \leqq \gamma(A \vee B) \delta(A \wedge B) \quad \text { for all } A, B \subset S,
$$

where $\alpha(A)=\sum(a \in A) \alpha(a)$ and $A \vee B=\{a \cup b ; a \in A, b \in B\}$ and $A \wedge B$ $=\{a \cap b ; a \in A, b \in B\}$.

Since every distributive lattice can be embedded in the subsets of some set we get an immediate

Corollary. If $S$ is a distributive lattice and (2) holds whenever $A, B$ each contain exactly one point of $S$ then (2) always holds. Here $S, A, B$ may be infinite.

Our theorem contains as special cases results of Anderson, Daykin, Fortuin, Ginibre, Greene, Holley, Kasteleyn, Kleitman, Seymour, West and others ${ }^{1}$. We discovered it whilst guests at the Mathematisches Forschungsinstitut Oberwolfach and thank all concerned for their kindness to us.

\section{The Proof}

Case $n=1$. Write 0,1 for $\phi,\{1\}$ respectively. The Conditions (1) are

$$
\alpha(0) \beta(0) \leqq \gamma(0) \delta(0)
$$

1 This is explained in detail in the forthcoming paper "Inequalities for a pair of maps $S \times S \rightarrow S$ with $S$ a finite set" by the same authors (submitted to Math. Zeitschrift). This paper contains several new inequalities also for other binary operations 


$$
\begin{aligned}
& \alpha(0) \beta(1) \leqq \gamma(1) \delta(0)=\varepsilon \quad \text { say } \\
& \alpha(1) \beta(0) \leqq \varepsilon \\
& \alpha(1) \beta(1) \leqq \gamma(1) \delta(1) .
\end{aligned}
$$

When $A$ and $B$ both contain two elements the result (2) becomes

$$
(\alpha(0)+\alpha(1))(\beta(0)+\beta(1)) \leqq(\gamma(0)+\gamma(1))(\delta(0)+\delta(1)) .
$$

Suppose $0<\varepsilon$ for otherwise (7) is trivial. We decrease $\gamma(0)$ and $\delta(1)$ until we get equality in (3) and (6). Then (7) simplifies to

$$
0 \leqq(\alpha(0) \beta(1)-\varepsilon)(\alpha(1) \beta(0)-\varepsilon) / \varepsilon,
$$

which holds by (4) and (5). The remaining choices for $A, B$ hold by inspection, so case $n=1$ is verified.

Induction Step. Assume the result for $n=m \geqq 1$ and consider the case $n=m+1$. Write $S, T, P$ for the family of all subsets of $\{1,2, \ldots, m+1\},\{2,3, \ldots, m+1\},\{1\}$ respectively.

Given $a \in S$ put $a^{*}=a \backslash\{1\} \in T$ and $* a=a \backslash\{2,3, \ldots, m+1\} \in P$. Let $\alpha, \beta, \gamma, \delta, A$, $B$ be chosen and fixed. Define $\alpha_{2}, \beta_{2}, \gamma_{2}, \delta_{2}$ on $T$ by

$$
\begin{aligned}
& \alpha_{2}(c)=\sum\left(a \in A, a^{*}=c\right) \alpha(a) \\
& \gamma_{2}(c)=\sum\left(a \in A \vee B, a^{*}=c\right) \gamma(a)
\end{aligned}
$$

with similar expressions for $\beta_{2}$ and $\delta_{2}$. Then

$$
\alpha(A)=\sum_{a \in A} \alpha(a)=\sum_{c \in T}\left(\sum_{\substack{a \in A \\ a^{*}=c}} \alpha(a)\right)=\sum_{c \in T} \alpha_{2}(c)=\alpha_{2}(T)
$$

and similarly

$$
\beta(B)=\beta_{2}(T), \quad \gamma(A \vee B)=\gamma_{2}(T), \quad \delta(A \wedge B)=\delta_{2}(T) .
$$

Assume for the moment that

$$
\alpha_{2}(c) \beta_{2}(d) \leqq \gamma_{2}(c \cup d) \delta_{2}(c \cap d) \quad \text { for all } c, d \in T
$$

Using $T \vee T=T$ and our induction hypothesis we get

$$
\alpha(A) \beta(B)=\alpha_{2}(T) \beta_{2}(T) \leqq \gamma_{2}(T \vee T) \delta_{2}(T \wedge T)=\gamma(A \vee B) \delta(A \wedge B)
$$

which is (2) as required. Hence it remains to prove (8).

Let $c, d \in T$ be fixed arbitrarily. Write $e=c \cup d$ and $f=c \cap d$. Define $\alpha_{1}, \beta_{1}, \gamma_{1}, \delta_{1}$ on $P$ by

$$
\begin{aligned}
& \alpha_{1}(p)= \begin{cases}\alpha(p \cup c) & \text { if } p \cup c \in A \\
0 & \text { otherwise }\end{cases} \\
& \beta_{1}(p)= \begin{cases}\beta(p \cup d) & \text { if } p \cup d \in B \\
0 & \text { otherwise }\end{cases}
\end{aligned}
$$




$$
\begin{aligned}
& \gamma_{1}(p)= \begin{cases}\gamma(p \cup e) & \text { if } p \cup e \in A \vee B \\
0 & \text { otherwise }\end{cases} \\
& \delta_{1}(p)= \begin{cases}\delta(p \cup f) & \text { if } p \cup f \in A \wedge B \\
0 & \text { otherwise }\end{cases}
\end{aligned}
$$

Then

$$
\alpha_{2}(c)=\sum_{\substack{a \in A \\ a^{*}=c}} \alpha(a)=\sum_{p \in P}\left(\sum_{\substack{a \in A \\ a^{*}=c \\ * a=p}} \alpha(a)\right)=\sum_{p \in P} \alpha_{1}(p)=\alpha_{1}(P)
$$

and similarly

$$
\beta_{2}(d)=\beta_{1}(P), \quad \gamma_{2}(e)=\gamma_{1}(P), \quad \delta_{2}(f)=\delta_{1}(P) .
$$

Assume for the moment that

$$
\alpha_{1}(p) \beta_{1}(q) \leqq \gamma_{1}(p \cup q) \delta_{1}(p \cap q) \quad \text { for all } p, q \in P .
$$

Then by the case $n=1$ we have

$$
\alpha_{1}(P) \beta_{1}(P) \leqq \gamma_{1}(P \vee P) \delta_{1}(P \wedge P),
$$

or in other words (8) holds. So it now remains for (9) to be proved.

The left hand side of (9) is zero unless $p \cup c \in A$ and $q \cup d \in B$ in which case it is $\alpha(p \cup c) \beta(a \cup d)$. We then have

$$
\begin{gathered}
(p \cup c) \cup(q \cup d)=(p \cup q) \cup e \in A \vee B \quad \text { and } \\
(p \cup c) \cap(q \cup d)=(p \cap q) \cup f \in A \wedge B .
\end{gathered}
$$

Hence the right hand side of (9) is $\gamma((p \cup q) \cup e) \delta((p \cap q) \cup f)$ so (9) holds by hypothesis (1) and Theorem 1 follows inductively.

\section{References}

1. Anderson, I.: Intersection theorems and a lemma of Kleitman. Discrete Math. (To appear)

2. Daykin, D.E.: A lattice is distributive iff $|A||B| \leqq|A \vee B||A \wedge B|$. Nanta Math. (To appear)

3. Daykin, D.E.: Poset functions commuting with the product and yielding Čebyčev type inequalities. C.N.R.S. Colloque, Paris (1976) (To appear)

4. Daykin, D.E., Kleitman, D.J., West. D.B.: The number of meets between two subsets of a lattice. J. Combinatorial Theory [submitted]

5. Greene, C., Kleitman, D.J.: Proof techniques in the theory of finite sets. M.A.A. Studies in Combinatorics. Editor G.C. Rota (To appear)

6. Fortuin, C.M., Kasteleyn, P.W., Ginibre, J.: Correlation inequalities on some partially ordered sets. Comm. Math. Phys. 22 89-103 (1971)

7. Holley, R.: Remarks on the FKG inequalities. Comm. Math. Phys. 36, 227-231 (1974)

8. Kleitman, D.J.: Families of non-disjoint subsets. J. Combinatorial Theory 1, 153-155 (1966)

9. Seymour, P.D.: On incomparable collections of sets. Mathematika Period. Sb. Pererodov Inostran Statei. 20, 208-209 (1973) 